\title{
Numerical study of mode conversion between lower hybrid and whistler waves on short-scale density striations
}

\author{
B. Eliasson ${ }^{1,2}$ and K. Papadopoulos ${ }^{3}$ \\ Received 4 April 2008; revised 26 May 2008; accepted 3 June 2008; published 17 September 2008.
}

[1] We present a theoretical and numerical study of linear mode conversion of lower hybrid waves interacting with short-scale density striations in the Earth's ionosphere. The efficiency of the conversion process is investigated for different sets of parameters such as the angle of incidence, the wavelength of the lower hybrid wave, and the size of the striation. It is found that the most efficient whistler generation occurs at a critical angle of incidence where the whistler waves are driven resonantly along the density striations, and when the product of the striation width and the wave number of the lower hybrid wave is of the order unity. It is suggested that whistlers generated as a byproduct of upper hybrid F-region ionospheric heating can be observed on the ground and by satellites. The generated whistlers could be important for the precipitation of energetic electrons in the radiation belts.

Citation: Eliasson, B., and K. Papadopoulos (2008), Numerical study of mode conversion between lower hybrid and whistler waves on short-scale density striations, J. Geophys. Res., 113, A09315, doi:10.1029/2008JA013261.

\section{Introduction}

[2] Whistler waves are ubiquitous in the Earth's ionosphere and magnetosphere where they propagate along magnetic field lines between the hemispheres. They contribute to the pitch angle scattering and precipitation of radiation belt electrons in the inner radiation belts [Inan and Bell, 1991; Inan et al., 2003; Abel and Thorne, 1998a, 1998b]. The whistlers are naturally generated by lightning in the equatorial zone. Their propagation speed varies with the frequency and gives rise to their characteristic descending tone. Recent satellite observations indicate that lightning-induced whistlers contribute to lower hybrid turbulence and ion heating in the equatorial ionosphere [Berthelier et al., 2008]. Whistlers are also generated at shocks in the magnetosphere and at the bow shock, and are thought to be important for fast magnetic reconnection [Deng and Matsumoto, 2001]. Observations with the CLUSTER satellites near the plasmapause revealed lowfrequency $(100-500 \mathrm{~Hz})$ whistlers correlated with density fluctuations and high-frequency $(3-6 \mathrm{kHz})$ whistlers anticorrelated with the density fluctuations [Moullard et al., 2002]. In the laboratory, whistlers have been observed to be guided along magnetic [Gushchin et al., 2005] and density [Zaboronkova et al., 1992] ducts. The channeling of whistlers along density troughs, crests and gradients has been studied in recent numerical studies [Streltsov et al., 2006]. In the presence of magnetic field aligned plasma irregular-

\footnotetext{
${ }^{1}$ Department of Physics, Umeå University, Umeå, Sweden.

${ }^{2}$ Theoretische Physik IV, Ruhr-Universität Bochum, Bochum, Germany.

${ }^{3}$ Departments of Physics and Astronomy, University of Maryland, College Park, Maryland, USA.
}

Copyright 2008 by the American Geophysical Union. 0148-0227/08/2008JA013261\$09.00 ities (striations), whistlers can be mode converted into lower hybrid waves and vice versa. At the Large Plasma Device (LAPD) at UCLA, it was demonstrated experimentally that lower hybrid waves can be generated by whistlers on density striations [Bamber et al., 1995; Rosenberg and Gekelman, 1998], and that lower hybrid waves interacting with density striations have their largest wave fields in the regions of steepest density gradient in the density striations [Rosenberg and Gekelman, 2000, 2001]. The generation of whistler waves by lower hybrid waves on density striations has been investigated theoretically for planar density irregularities [Bell and Ngo, 1990], where the possibility of generating whistlers by lower hybrid waves also was studied. In experiments at Arecibo, it was demonstrated that VLF signals can couple into ionospheric ducts and propagate into the conjugate hemisphere as ducted whistlers, where they can parametrically excite lower hybrid waves [Lee and Kuo, 1984]. The generation of whistlers by $\mathrm{HF}$ induced lower hybrid waves in the presence of density striations has been investigated theoretically [Borisov, 1995]. During ionospheric heating experiments at the Sura facility near Nizny Novgorod, Russia, whistlers were observed on the top side ionosphere by the Intercosmos-24 satellite [Vas'kov et al., 1998]. Ionospheric density striations created by HF waves can have sizes ranging from a fraction of a meter up to 10-m scale [Thome and Blood, 1974; Minkoff et al., 1974a, 1974b; Minkoff, 1974; Djuth et al., 1985; Kelley et al., 1995].

[3] In the present paper we investigate the generation of low-frequency (in comparison to the electron gyrofrequency) whistler waves by lower hybrid waves interacting with short-scale (in comparison with the whistler wavelength) density striations. The paper focuses on the efficiency of the whistler generation as a function of different 
parameters, and the interplay between the whistlers and lower hybrid waves in the presence of a collection of density striations. In section 2 , we derive the mathematical model that governs the dynamics of the interaction between lower hybrid and whistler waves in the presence of density striations/cavities. The dependence of the efficiency on the angle of incidence for mode conversion of lower hybrid waves into whistlers in the presence of striations is derived in section 3 . In section 4 , we investigate numerically the efficiency of whistler generation for different parameters, as well as the interplay between whistler and lower hybrid generation in the presence of several striations. Finally, conclusions are presented in section 5 .

\section{Mathematical Model}

[4] We derive next the governing equations for coupled lower hybrid and whistler waves in the presence of density striations. We assume that the ions are unmagnetized since the lower hybrid and whistler frequencies are much higher than the ion gyrofrequency, and that that quasi-neutrality applies.

[5] The electromagnetic field is governed by Faraday's and Ampère's equations,

$$
\frac{\partial \mathbf{B}}{\partial t}=-\nabla \times \mathbf{E}
$$

and

$$
\nabla \times \mathbf{B}=\mu_{0} e \tilde{n}\left(\mathbf{v}_{i 1}-\mathbf{v}_{e 1}\right)
$$

respectively, where $\mu_{0}$ is the magnetic permeability in vacuum and $e$ is the magnitude of the electron charge. Here $\tilde{n}(\mathbf{x})=n_{0}+n_{\text {str }}(\mathbf{x})$ is the zero-order background electron number density, which is composed of the equilibrium density $n_{0}$ and the "striation density" $n_{\text {str }}(\mathbf{x})$. We have neglected the displacement current in equation (2) since the phase speed of the lower hybrid and whistler waves are much smaller than the speed of light.

[6] We shall for simplicity consider cold electrons and ions $T_{e}=T_{i}=0$, which is valid for wavelengths much longer than the particle's Debye length. The ion dynamics is governed by the linearized ion continuity equation equations

$$
\frac{\partial n_{i 1}}{\partial t}+\nabla \cdot(\tilde{n} \mathbf{v} i)=0
$$

and the unmagnetized ion momentum equation

$$
m_{i} \tilde{n} \frac{\partial \mathbf{v} i}{\partial t}=e \tilde{n} \mathbf{E}
$$

where $m_{i}$ is the ion mass. The electrons are governed by the continuity equation

$$
\frac{\partial n_{e 1}}{\partial t}+\nabla \cdot\left(\tilde{n} \mathbf{v}_{e 1}\right)=0
$$

and momentum equation

$$
m_{e} \tilde{n} \frac{\partial \mathbf{v}_{e 1}}{\partial t}=-e \tilde{n} \mathbf{E}-e \tilde{n} \mathbf{v}_{e 1} \times \mathbf{B}_{0}
$$

where $m_{e}$ is the electron mass, and $\mathbf{B}_{0}=B_{0} \hat{\mathbf{z}}$ is the geomagnetic field directed in the $z$ direction. The assumption of quasineutrality $n_{e 1}=n_{i 1} \equiv n_{1}$ combined with the continuity equations leads to the condition

$$
\nabla \cdot\left(\tilde{n} \mathbf{v}_{i 1}\right)=\nabla \cdot\left(\tilde{n} \mathbf{v}_{e 1}\right)
$$

for the velocity fields, and we have the continuity equation

$$
\frac{\partial n_{1}}{\partial t}+\nabla \cdot\left(\tilde{n} \mathbf{v}_{e 1}\right)=0
$$

It is now convenient to introduce the ion and electron particle current fields $\mathbf{j}_{i 1}=\tilde{n} \mathbf{v}_{i 1}$ and $\mathbf{j}_{e 1}=\tilde{n} \mathbf{v}_{e 1}$, respectively. Then equations $(2)-(8)$ yield

$$
\begin{gathered}
\nabla \times \mathbf{B}=\mu_{0} e\left(\mathbf{j}_{i 1}-\mathbf{j}_{e 1}\right), \\
m_{i} \frac{\partial \mathbf{j}_{i 1}}{\partial t}=e \tilde{n} \mathbf{E}
\end{gathered}
$$

$$
m_{e} \frac{\partial \mathbf{j}_{e 1}}{\partial t}=-e \tilde{n} \mathbf{E}-e \mathbf{j}_{e 1} \times \mathbf{B}_{0}
$$

$$
\nabla \cdot \mathbf{j}_{i 1}=\nabla \cdot \mathbf{j}_{e 1}
$$

and

$$
\frac{\partial n_{1}}{\partial t}+\nabla \cdot \mathbf{j}_{e 1}=0
$$

The mode coupling between the lower hybrid and whistler waves is mediated by the $\tilde{n} \mathbf{E}$-terms in the right-hand sides of equations (10) and (11).

[7] In order to cast the governing equations into a numerically more convenient form, we now add equations (10) and (11), take the divergence of the resulting equation, and use equation (12) to eliminate $\nabla \cdot \mathbf{j}_{i 1}$. The result is

$$
\frac{\partial}{\partial t} \nabla \cdot \mathbf{j}_{e 1}=-\frac{e}{m_{i}} \nabla \cdot\left(\mathbf{j}_{e 1} \times \mathbf{B}_{0}\right)
$$

where we used $m_{i} \gg m_{e}$. On the other hand, taking the double curl of equation (11) yields

$$
\frac{\partial}{\partial t}\left[\nabla\left(\nabla \cdot \mathbf{j}_{e 1}\right)-\nabla^{2} \mathbf{j}_{e 1}\right]=-\frac{e}{m_{e}} \nabla \times\left[\nabla \times\left(\tilde{n} \mathbf{E}+\mathbf{j}_{e 1} \times \mathbf{B}_{0}\right)\right]
$$

where we used the vector identity $\nabla \times\left(\nabla \times \mathbf{j}_{e 1}\right)=$ $\nabla\left(\nabla \cdot \mathbf{j}_{e 1}\right)-\nabla^{2} \mathbf{j}_{e 1}$. Taking the gradient of equation (14) and subtracting the result from equation (15) yields

$$
\begin{aligned}
-\frac{\partial}{\partial t} \nabla^{2} \mathbf{j}_{e 1}= & -\frac{e}{m_{e}} \nabla \times\left[\nabla \times\left(\tilde{n} \mathbf{E}+\mathbf{j}_{e 1} \times \mathbf{B}_{0}\right)\right] \\
& +\frac{e}{m_{i}} \nabla\left[\nabla \cdot\left(\mathbf{j}_{e 1} \times \mathbf{B}_{0}\right)\right]
\end{aligned}
$$


We now use that $\nabla \times(\tilde{n} \mathbf{E})=\nabla \times\left(n_{\mathrm{str}} \mathbf{E}\right)+n_{0} \nabla \times \mathbf{E}=\nabla \times$ $\left(n_{\mathrm{str}} \mathbf{E}\right)-n_{0} \partial \mathbf{B} / \partial t$, to obtain

$$
\begin{aligned}
l \frac{\partial}{\partial t}\left(\nabla^{2} \mathbf{j}_{e 1}+\frac{e n_{0}}{m_{e}} \nabla \times \mathbf{B}\right)= & \frac{e}{m_{e}} \nabla \times\left[\nabla \times\left(n_{s t r} \mathbf{E}+\mathbf{j}_{e 1} \times \mathbf{B}_{0}\right)\right] \\
& -\frac{e}{m_{i}} \nabla\left[\nabla \cdot\left(\mathbf{j}_{e 1} \times \mathbf{B}_{0}\right)\right] .
\end{aligned}
$$

The magnetic field is computed from the divergence free part of the electron current in equation (9), as $\nabla \times \mathbf{B}=$ $\mu_{0} e \nabla^{-2}\left[\nabla\left(\nabla \cdot \mathbf{j}_{e 1}\right)-\nabla^{2} \mathbf{j}_{e 1}\right]$, so that equation (17) takes the form

$$
\begin{aligned}
\frac{\partial}{\partial t}\left(\lambda_{e}^{2} \nabla^{2}-1\right) \mathbf{j}_{e 1}= & \frac{e \lambda_{e}^{2}}{m_{e}} \nabla \times\left[\nabla \times\left(n_{s t r} \mathbf{E}+\mathbf{j}_{e 1} \times \mathbf{B}_{0}\right)\right] \\
& -\frac{e}{m_{i}} \nabla^{-2}\left(\lambda_{e}^{2} \nabla^{2}-1\right) \nabla\left[\nabla \cdot\left(\mathbf{j}_{e 1} \times \mathbf{B}_{0}\right)\right],
\end{aligned}
$$

where we used equation (14) to eliminate the $\nabla \cdot \mathbf{j}_{e}$ term, and where $\lambda_{e}=c / \omega_{p e}$ is the electron skin depth, $c$ is the speed of light in vacuum, $\omega_{p e}=\left(n_{0} e^{2} / \varepsilon_{0} m_{e}\right)^{1 / 2}$ is the electron plasma frequency, and $\varepsilon_{0}$ is the electric permittivity in vacuum. The $\nabla^{-2}$ operator corresponds to $k^{-2}$ in Fourier space, where $k^{2}=k_{y}^{2}+k_{z}^{2}$, and where $k_{y}$ and $k_{z}$ are the $y$ and $z$ components of the wavevector. In the pseudospectral method used in the numerical simulations below, we set the Fourier components corresponding to $k=0$ to zero in the numerical approximation of $\nabla^{-2}$.

[8] In order to calculate the beating between the lower hybrid waves and the striations in the $n_{s t r} \mathbf{E}$-term, we need to determine the electric field. The curl of the electric field is given by equation (1), and we also need the divergence of the electric field to determine it completely. Taking the divergence of equation (10) with $\tilde{n} \approx n_{0}$ and noting that $\partial \nabla \cdot \mathbf{j}_{\mathbf{i} 1} / \partial t=\partial \nabla \cdot \mathbf{j}_{\mathbf{e} 1} / \partial t$, we replace the time derivative with the right-hand side of equation (14) to obtain

$$
\nabla \cdot \mathbf{E} \simeq-\frac{\nabla \cdot\left(\mathbf{j}_{e 1} \times \mathbf{B}_{0}\right)}{n_{0}},
$$

where we used that $m_{i} \gg m_{e}$. Using the vector identity $\nabla^{2} \mathbf{E}=\nabla(\nabla \cdot \mathbf{E})-\nabla \times(\nabla \times \mathbf{E})$ together with equations (1) and (19) we obtain

$$
\nabla^{2} \mathbf{E} \simeq-\frac{\nabla\left[\nabla \cdot\left(\mathbf{j}_{e 1} \times \mathbf{B}_{0}\right)\right]}{n_{0}}+\nabla \times \frac{\partial \mathbf{B}}{\partial t} .
$$

Taking the curl of equation (9) with $\left|\nabla \times \mathbf{j}_{i 1}\right| \ll\left|\nabla \times \mathbf{j}_{e 1}\right|$ and $\nabla \cdot \mathbf{B}=0$ yields $\nabla^{2} \mathbf{B}=\mu_{0} e \nabla \times \mathbf{j}_{e 1}$. Solving for $\mathbf{B}$, inserting the result into (20), and eliminating the time derivative with the help of equation (18), gives the electric field as

$$
\begin{aligned}
\nabla^{2} \mathbf{E} \simeq & -\frac{\nabla\left[\nabla \cdot\left(\mathbf{j}_{e 1} \times \mathbf{B}_{0}\right)\right]}{n_{0}}-\frac{1}{n_{0}}\left(\lambda_{e}^{2} \nabla^{2}-1\right)^{-1} \nabla \\
& \times\left[\nabla \times\left(\mathbf{j}_{e 1} \times \mathbf{B}_{0}\right)\right] .
\end{aligned}
$$

Equation (18), supplemented by equation (21), governs the interaction and mode conversion between lower hybrid and whistler waves in the presence of density striations.
[9] It is convenient to simplify equation (18) by separating it into one equation for electrostatic lower hybrid waves and one for whistler waves, which are coupled via the striation. In doing so, we decompose the electron particle current density as $\mathbf{j}_{e 1}=\mathbf{j}_{L H}+\mathbf{j}_{W}$ and the electric field as $\mathbf{E}=$ $\mathbf{E}_{L H}+\mathbf{E}_{W}$ where the subscripts $L H$ and $W$ denotes "lower hybrid" and "whistler", respectively. The lower hybrid waves are almost completely electrostatic and have wave their vectors almost perpendicular to the magnetic field lines. The short wavelength $\left(\left|\lambda_{e}^{2} \nabla^{2}\right| \gg 1\right)$ lower hybrid evolution equation is obtained from equation (18) as

$$
\begin{aligned}
\frac{\partial \mathbf{j}_{L H}}{\partial t}= & \nabla^{-2}\left\{\frac{e}{m_{e}} \nabla \times\left[\nabla \times\left(n_{s t r} \mathbf{E}_{W}+\mathbf{j}_{L H} \times \mathbf{B}_{0}\right)\right]\right. \\
& \left.-\frac{e}{m_{i}} \nabla\left[\nabla \cdot\left(\mathbf{j}_{L H} \times \mathbf{B}_{0}\right)\right]\right\},
\end{aligned}
$$

where the $n_{s t r} \mathbf{E}_{W}$ term represents the coupling of the whistler electric field to the lower hybrid waves via the density striation. On the other hand, for the whistler waves we neglect the influence of the ions due to their large mass, so that the whistler evolution equation is obtained from equation (18) as

$$
\frac{\partial \mathbf{j}_{W}}{\partial t}=-\frac{e \lambda_{e}^{2}}{m_{e}}\left(1-\lambda_{e}^{2} \nabla^{2}\right)^{-1} \nabla \times\left[\nabla \times\left(n_{s t r} \mathbf{E}_{L H}+\mathbf{j}_{W} \times \mathbf{B}_{0}\right)\right],
$$

where the $n_{s t r} \mathbf{E}_{L H}$ term couples the lower hybrid electric field to the whistler waves via the density striation. The lowfrequency, long-wavelength whistlers are characterized by $\left|\lambda_{e}^{2} \nabla^{2}\right| \ll 1$, but we keep the $\lambda_{e}^{2} \nabla^{2}$ term in equation (23) for numerical convenience since it limits the whistler frequency to the electron gyrofrequency at short wavelengths. For $n_{\text {str }}=0$, equations (22) and (23) are decoupled and we have pure whistler and lower hybrid waves. The electrostatic field of the lower hybrid wave is obtained from equation (21) as

$$
\mathbf{E}_{L H}=-\frac{1}{n_{0}} \nabla \nabla^{-2}\left[\nabla \cdot\left(\mathbf{j}_{L H} \times \mathbf{B}_{0}\right)\right],
$$

while the whistler electric field is in the long wavelength limit $\left|\lambda_{e}^{2} \nabla^{2}\right| \ll 1$ obtained as

$$
\mathbf{E}_{W}=-\frac{1}{n_{0}} \mathbf{j}_{W} \times \mathbf{B}_{0} .
$$

Equations (22)-(25) are the desired set of equations for the mode conversion between lower hybrid and whistler waves in the presence of density striations.

\section{Condition for Resonant Mode Conversion}

[10] The most efficient mode conversion of lower hybrid waves into parallel (to the magnetic field lines) propagating whistlers is likely to occur when there is a simultaneous matching between the frequencies and the parallel components of the wave numbers of the lower hybrid waves and the whistler waves, so that the whistler waves are driven resonantly along the magnetic field aligned density stria- 

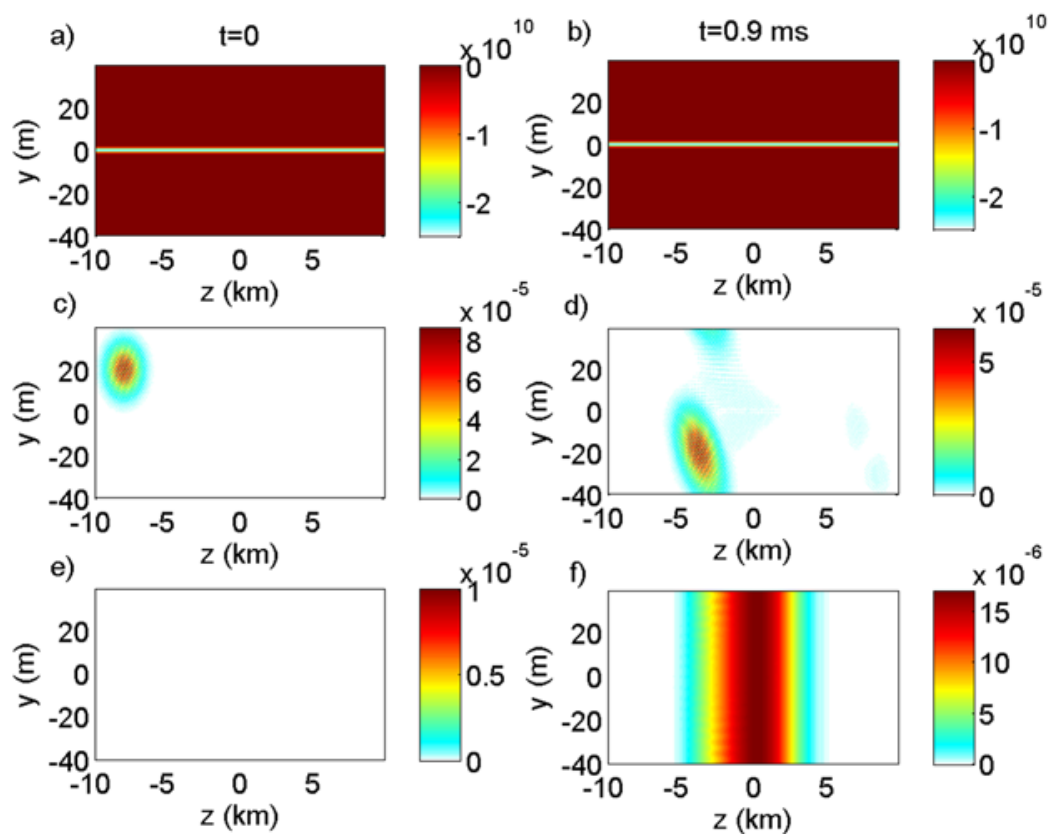

Figure 1. Generation of whistler waves by lower hybrid waves in the presence of a density striation, initial condition $t=0$ (left), and at $t=0.9 \mathrm{~ms}$ (right). The striation $n_{\mathrm{str}}(\mathrm{a}, \mathrm{b})$ has the width $D_{\mathrm{str}}=1 \mathrm{~m}$, has a maximum density depletion of $5 \%$ of the background density, and is aligned with the geomagnetic field lines. The $y$ component of the electric field (c, d) shows the lower hybrid wave with the wave number $k=1.5 \mathrm{~m}^{-1}$ and the angle of incidence given by $\cos (\theta)=0.093$. The lower hybrid wave envelope is initially centered at $y=20 \mathrm{~m}, z=-8 \mathrm{~km}(\mathrm{c})$. At $t=0.9 \mathrm{~ms}(\mathrm{~d})$, it has past over the striation and has reached $y=-15 \mathrm{~m}, z \simeq-3 \mathrm{~km}$. The amplitude of the whistler magnetic field, $c|\mathbf{B}|$, is shown in Figures $1 \mathrm{e}$ and 1f. The whistler waves have a wavelength of $\sim 450 \mathrm{~m}$ and is propagating along the magnetic field lines in the rightward direction.

tions. The dispersion relation for lower hybrid waves in the electrostatic limit $\left(\lambda_{e}^{2} k^{2} \gg 1\right)$ is given by

$$
\omega^{2}=\frac{\omega_{c e} \omega_{c i} k_{\perp}^{2}+\omega_{c e}^{2} k_{z}^{2}}{k_{\perp}^{2}+k_{z}^{2}},
$$

while that of low-frequency whistler waves $\left(\lambda_{e}^{2} k^{2} \ll 1\right)$, propagating parallel to the magnetic field lines, is given by

$$
\omega=\frac{c^{2} k_{z}^{2}}{\omega_{p e}^{2}} \omega_{c e} .
$$

Using $k_{z}=k \cos (\theta)$ and $k_{\perp}=k \sin (\theta)$, we obtain from equations (26) and (27)

$$
\cos ^{2}(\theta)=\frac{1}{2} \frac{\omega_{p e}^{4}}{c^{4} k^{4}}+\sqrt{\frac{1}{4} \frac{\omega_{p e}^{8}}{c^{8} k^{8}}+\frac{m_{e} \omega_{p e}^{4}}{m_{i} c^{4} k^{4}}}
$$

We note that equation (28) is independent of the magnetic field.

\section{Numerical Results}

[11] In order to study the coupling between lower hybrid and whistler waves in the presence of magnetic field aligned density striations, equations (22)-(25) are solved numerically. We use a rectangular simulation box with periodic boundary conditions. The box size is $L_{y} \times L_{z}=80 \times 10000$ m, and we use typically $N_{y} \times N_{z}=300 \times 150$ grid points to resolve the solution, so that the grid sizes become $\Delta y=$ $L_{y} / N_{\mathrm{y}} \approx 0.27 \mathrm{~m}$ and $\Delta z=L_{z} / N_{z} \approx 67 \mathrm{~m}$. The box sizes $L_{y}$ and $L_{z}$ have been chosen much larger than the wavelengths in the $y$ and $z$ direction, respectively, to avoid finite box size effects. The criterium for choosing grid sizes is that one must have more than two grid points per wavelength to represent the solution on the numerical grid. Hence in the $z$ dimension we must have $k_{z}<\pi / \Delta z$, and in the $y$ dimension we must also take into account the mixing between the wave and the striation (the $n_{s t r} E_{L H}$ and $n_{s t r}$ $E_{W}$ terms) so that the condition becomes $k_{y}+k_{\text {str }} \ll \pi / \Delta y=$ $\pi N_{y} / L_{y}$ to avoid aliasing effects, where $k_{\text {str }}=1 / D_{\text {str }}$ is the spectral width of the striation. The grid size is in some cases decreased to ensure that the solution is resolved on the numerical grid. A pseudo-spectral method is used to approximate the spatial derivatives, and a Runge-Kutta scheme is used to advance the solution in time, with the time step $\Delta t=3 \times 10^{-7} \mathrm{~s}$.

[12] For concreteness we use parameters of the ionospheric $\mathrm{F}$ region given by $n_{0}=5 \times 10^{11} \mathrm{~m}^{-3}, m_{i} / m_{e}=$ 29500 (oxygen ions) and $B_{0}=4.8 \times 10^{-5} \mathrm{~T}$. The magnetic field $\mathbf{B}_{0}=B_{0} \hat{\mathbf{z}}$ is aligned with the $z$ axis. The electron plasma frequency is $\omega_{p e}=4 \times 10^{7} \mathrm{~s}^{-1}$ and the electron gyrofrequency is $\omega_{c e}=8.44 \times 10^{6} \mathrm{~s}^{-1}$.

[13] Figure 1 shows the time evolution of a lower hybrid wave interacting with one density striation, and the gener- 

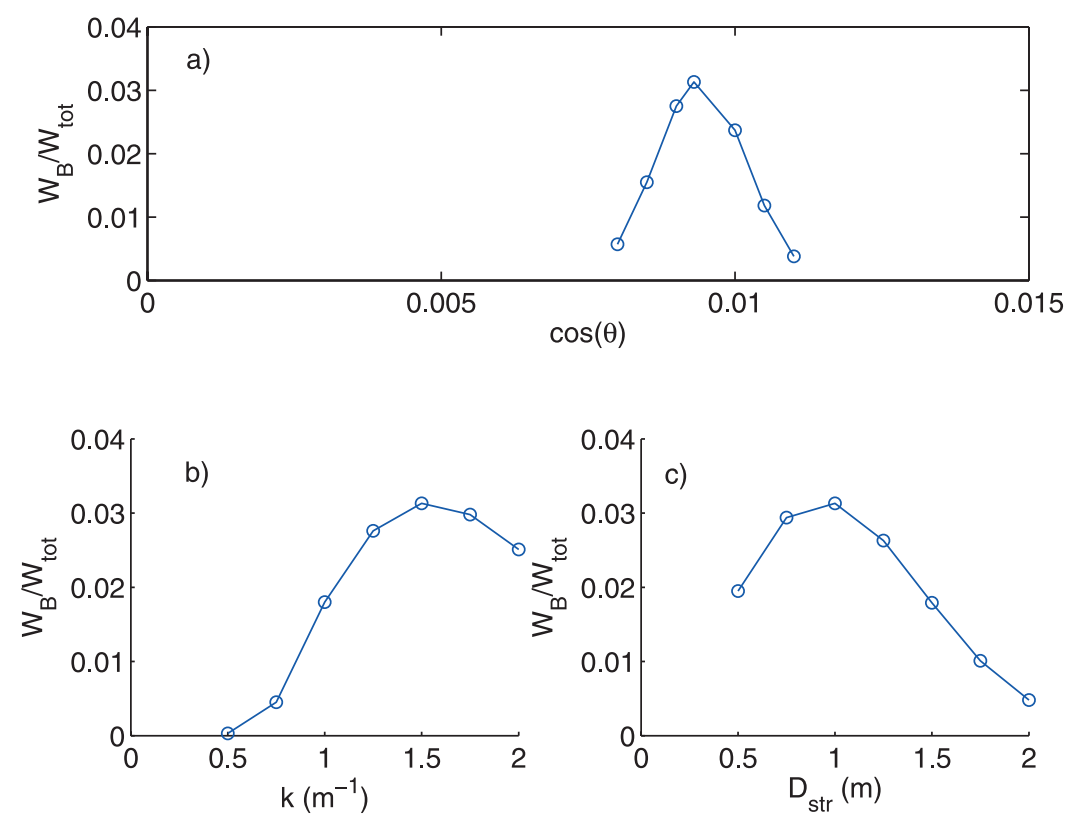

Figure 2. The efficiency of whistler generation (generated whistler energy divided by total energy) as a function of (a) $\cos (\theta)=k_{z} / k$ with $k=1.5 \mathrm{~m}^{-1}$ and $D_{s t r}=1 \mathrm{~m}$, (b) of $k$ with $D_{s t r}=1 \mathrm{~m}$, and (c) of $D_{\text {str }}$ with $k=1.5 \mathrm{~m}^{-1}$. The circles indicate measured values. The plots in Figures $2 \mathrm{~b}$ and $2 \mathrm{c}$ are done with the resonant angle of incidence given by equation (28).

ation of whistler waves. The striation density, which is aligned with the $z$ axis, is given by

$$
n_{s t r}=-n_{0, s t r} \exp \left(-\frac{y^{2}}{D_{s t r}^{2}}\right),
$$

where $D_{s t r}$ is the width of the striation, and $n_{0, s t r}$ is the magnitude of the density striation; we use $n_{0, \text { str }}=0.25 \times$ $10^{11} \mathrm{~m}^{-3}$ (corresponding to $5 \%$ of the background number density $n_{0}$ ). The striation width is set to $D_{\text {str }}=1 \mathrm{~m}$. As initial conditions for our simulation, we use

$$
\begin{gathered}
j_{1 x}=j_{0}(y, z) \frac{\omega_{c e}}{\omega} \sin \left(k_{y} y+k_{z} z\right), \\
j_{1 y}=j_{0}(y, z) \cos \left(k_{y} y+k_{z} z\right),
\end{gathered}
$$

and

$$
j_{1 z}=-j_{0}(y, z) \frac{\left(\omega_{c e}^{2}-\omega^{2}\right)}{\omega^{2}} \frac{k_{z}}{k_{y}} \cos \left(k_{y} y+k_{z} z\right),
$$

where the frequency $\omega$ is given by the lower hybrid dispersion relation (equation (26)), and the amplitude of the wave packet is given as a Gaussian envelope

$$
j_{0}(y, z)=10^{10} \exp \left[-\frac{(y-20)^{2}}{D_{y}^{2}}-\frac{(z+8000)^{2}}{D_{z}^{2}}\right],
$$

where the pulse widths are taken to be $D_{y}=10 \mathrm{~m}$ and $D_{z}=$ $1000 \mathrm{~m}$ in the $y$ and $z$ directions, respectively. For the lower hybrid wave, we use the wave number $k=1.5 \mathrm{~m}^{-1}$ and the resonance condition (equation (28)) for the angle, yielding $\cos (\theta)=0.0093, k_{\perp} \equiv k_{y}=\approx 1.5 \mathrm{~m}^{-1}$ and $k_{z} \approx 0.0138 \mathrm{~m}^{-1}$. We see in Figure 1 that the lower hybrid wave packet, initially centered at $y=20 \mathrm{~m}$ and $z=-8 \mathrm{~km}$ propagates obliquely (perpendicularly to the wave number) and crosses the striation centered at $y=0$. At $t=0.85 \mathrm{~ms}$, it has traversed the striation and has reached $y=-15 \mathrm{~m}, z \simeq-3 \mathrm{~km}$. During the interaction between the lower hybrid wave and the striation, whistlers have been excited and are propagating along the magnetic field lines in the rightward direction (see Figure 1f). The whistler waves have a wavelength of $2 \pi / k_{z} \approx 450 \mathrm{~m}$.

[14] In Figure 2, we repeat the numerical experiment of Figure 1 and allow the lower wave packet to propagate and cross the striation for several sets of parameters. At the end of the simulation, we measure the total magnetic energy of the whistler wave, and calculate the efficiency of the energy conversion from the lower hybrid to the whistler wave. We define the conversion efficiency as the ratio between the magnetic (whistler) energy $W_{B}$ and the total energy of the lower hybrid wave packet, $W_{\text {tot }}=W_{e}+W_{I}+W_{B}$, where $W_{e}$ and $W_{i}$ are the kinetic energy of the electrons and ions, respectively. The energies are obtained as

$$
\begin{gathered}
W_{B}=\int \frac{B^{2}}{2 \mu_{0}} d^{2} x \simeq \frac{m_{e}}{2 \lambda_{e}^{2} n_{0}}\left(\nabla^{-2} \nabla \times j_{e 1}\right)^{2} d^{2} x, \\
W_{e}=n_{0} \int \frac{m_{e} v_{e}^{2}}{2} d^{2} x=\frac{m_{e}}{2 n_{0}} \int j_{e 1}^{2} d^{2} x,
\end{gathered}
$$

and

$$
W_{i}=n_{0} \int \frac{m_{i} v_{i}^{2}}{2} d^{2} x \simeq \frac{m_{i}}{2 n_{0}} \int\left[\nabla^{-2} \nabla\left(\nabla \cdot \mathbf{j}_{e 1}\right)\right]^{2} d^{2} x
$$

where the integrals are taken over the simulation box. We use the same parameters as in Figure 1, except for the parameters that are varied as described below. In Figure 2a, the wave number is set to $2 \mathrm{~m}^{-1}$ and the angle of incidence $\theta$ is varied. Here, equation (28) predicts that there will be 

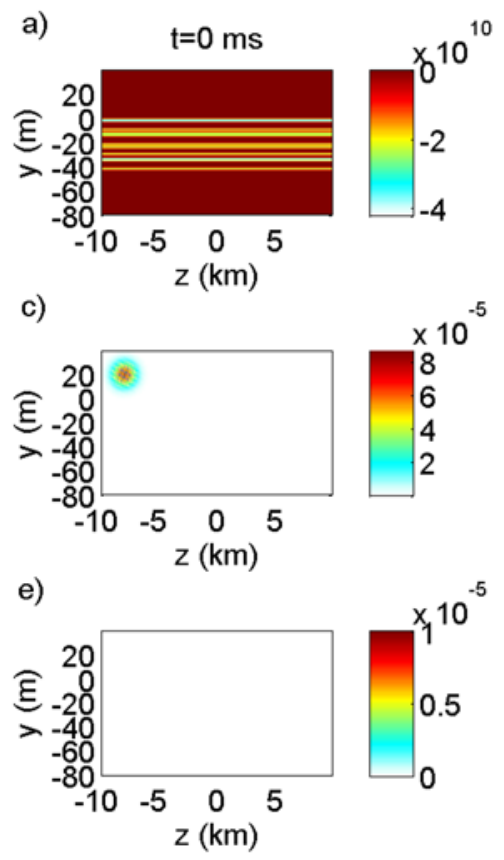
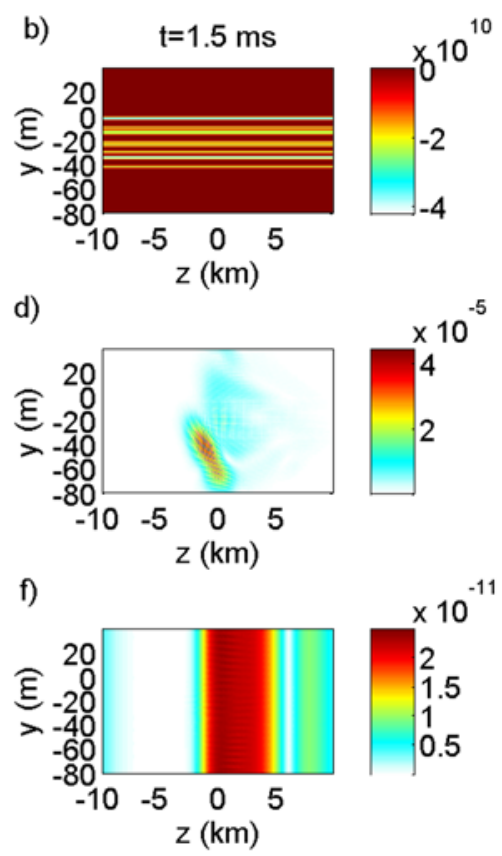

Figure 3. Generation of whistler waves by lower hybrid waves in the presence of a collection of density striations, initial condition $t=0$ (left), and at $t=1.5 \mathrm{~ms}$ (right). The 11 striations (a,b) have a width of $D_{\text {str }}=1 \mathrm{~m}$ and an amplitude of $5 \%$ of the background density, and are randomly spaced and centered at $y=-41.88 \mathrm{~m}, y=-34.63 \mathrm{~m}, y=-33.86 \mathrm{~m}, y=-29.83 \mathrm{~m}, y=-24.19 \mathrm{~m}, y=-21.82 \mathrm{~m}, y=$ $-14.39 \mathrm{~m}, y=-12.53 \mathrm{~m}, y=-9.65 \mathrm{~m}, y=-2.51 \mathrm{~m}$, and $y=-1.62 \mathrm{~m}$. The initial parameters for the lower hybrid wave are the same as in Figure 1. The $y$ component of the electric field (c, d) shows the lower hybrid waves at $t=0$, centered at $y=20 \mathrm{~m}, z=-8 \mathrm{~km}$ (c), and at $t=1.5 \mathrm{~ms}$ (d). The amplitude of the whistler wave magnetic field $c|\mathbf{B}|$ is shown in Figures $3 \mathrm{e}$ and $3 \mathrm{f}$.

resonant mode conversion for $\cos (\theta)=0.0093$, and that the generated whistler waves will have a wave number $k_{z}=$ $0.0138 \mathrm{~m}^{-1}$ corresponding to a wavelength of $\sim 450 \mathrm{~m}$. We see in Figure 2a that the maximum efficiency is $\sim 3 \%$, and that the efficiency is strongly peaked at $\cos (\theta)=0.0093$ as predicted by equation (28). In Figure $2 b$, we vary the wave number of the lower hybrid wave, while keeping the striation width $D_{s t r}=1 \mathrm{~m}$ constant. We see that there is an optimal conversion of lower hybrid waves when $k D_{\text {str }} \approx 1.5$. In Figure 2c, we vary the striation width $D_{\text {str }}$ for a fixed value of $k=1.5 \mathrm{~m}^{-1}$ and observe that there is a maximum conversion efficiency at $D_{s t r}=1 \mathrm{~m}$. The results of Figures $2 \mathrm{~b}$ and $2 \mathrm{c}$ indicate that there is a maximum generation of whistler waves for $D_{s t r} k \approx 1.5$.

[15] The interaction of the lower hybrid wave packet with a collection of striations is studied in Figures 3 and 4. The box size used is $L_{y} \times L_{z}=120 \times 10,000 \mathrm{~m}$, and we used $N_{y} \times N_{z}=300 \times 200$ grid points to resolve the solution. Here, there are 11 striations randomly spaced between $z \sim-40 \mathrm{~m}$ and $z \sim 0$; see Figure 3. The parameters are the same as in Figure 1, where the wave number $k=1.5 \mathrm{~m}^{-1}$ of the lower hybrid wave, together with the resonance condition (equation (28)), gives $\cos (\theta)=0.0093$ for the angle of incidence. In the simulation, we observe that the lower hybrid waves initially interact with the density striations to excite whistler waves. The whistler waves, on the other hand, generate lower hybrid waves in the interaction with the density striation. At the end of the simulation, exhibited in

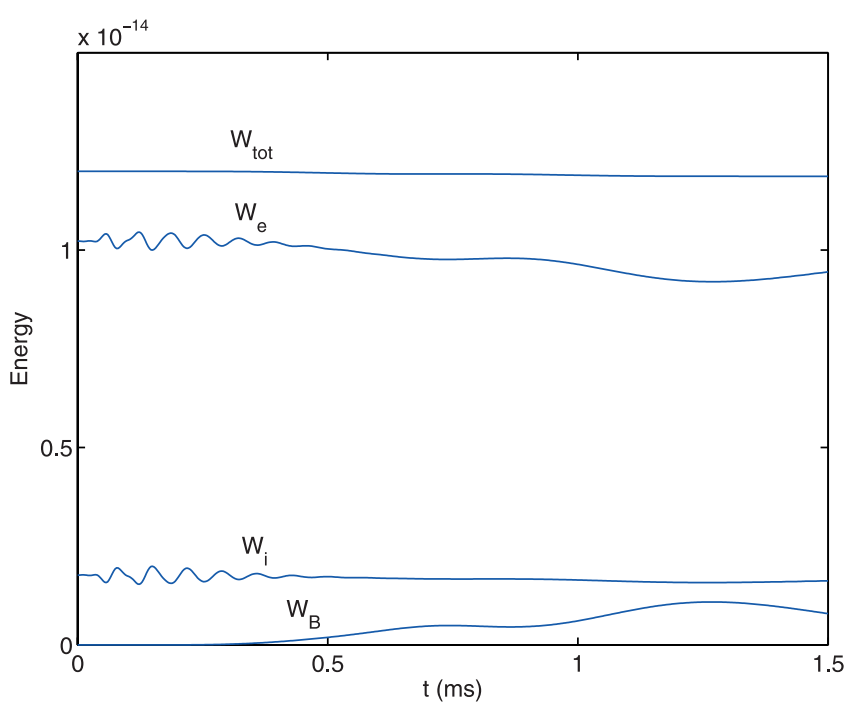

Figure 4. The particle kinetic energies associated with the lower hybrid oscillations $W_{e}$ and $W_{i}$, and the whistler wave magnetic energy $W_{B}$ as a function of time, for the simulation in Figure 3. The total energy $W_{t o t}=W_{e}+W_{i}+W_{B}$ is almost constant throughout the simulation. Energy is initially $(t<1.25 \mathrm{~ms})$ converted from lower hybrid wave into magnetic energy of the whistler wave, but at later times $(t>1.25 \mathrm{~ms})$ some of the whistler wave energy is converted back to lower hybrid wave energy. 
the right of Figure 3, we see that lower hybrid waves have been excited over a large region in space.

[16] In Figure 4, we have plotted the energies of the lower hybrid and whistler waves. Initially there is an efficient excitation of whistler waves, and the magnetic energy reaches about $10 \%$ of the total energy. At later times, the whistler energy is returned back to the lower hybrid waves and the whistler energy decreases to about $7 \%$ of the total energy at the end of the simulation. Hence short-scale density striations are not only important for the generation of whistlers by lower hybrid waves, but also for the absorption of whistler waves and the generation of lower hybrid waves. This was also observed in laboratory experiments at LAPD by Rosenberg and Gekelman [1998] who studied the mode conversion of incident whistler waves into lower hybrid waves on a single striation. In the laboratory experiment, the width of the striation was 3-4 times the lower hybrid wave length, which corresponds roughly to the most favorable case of mode conversion found here (see Figures $2 b$ and $2 c$ ).

\section{Discussion}

[17] We have developed a simple model for the mode conversion of lower hybrid waves into whistler waves in the presence of density striations. On the basis of the numerical results, we have found that there is a critical angle of incidence of the lower hybrid waves to the striation, where the whistler waves are driven resonantly and there is a maximum efficiency of whistler generation. Furthermore, the whistler wave generation is most efficient when the product of the striation width and the wave number of the lower hybrid wave is of the order unity. Typical efficiencies of whistler wave generation (whistler energy divided by total energy) is a few percent. In the presence of a collection of density striations, there is an efficient generation of whistler waves by lower hybrid waves, and also an efficient absorption of whistler waves and re-generation of lower hybrid waves. Hence in the presence of short-scale density striations in the Earth's ionosphere and magnetosphere, whistlers may be absorbed and prevented to propagate over large distances. The investigation has relevance for forthcoming ionospheric heating experiments with HAARP, in which striations and lower hybrid waves are generated at the upper hybrid layer, and where whistler waves are expected to be generated by the interaction between lower hybrid waves and the density striations. The whistler waves could be useful for the pitch angle scattering and precipitation of energetic electrons in the Earth's radiation belts. The detection of whistlers can be done on the ground and by overflying spacecrafts.

[18] Acknowledgments. This work was partially supported by the Swedish Research Council (VR) and by ONR MURI N00014-07-1-0789. B. E. acknowledges the support and hospitality of University of Maryland where this work was carried out.

[19] Amitava Bhattacharjee thanks the reviewers for their assistance in evaluating this paper.

\section{References}

Abel, B., and R. M. Thorne (1998a), Electron scattering loss in Earth's inner magnetosphere: 1. Dominant physical processes, J. Geophys. Res., 103(A2), 2385-2396.
Abel, B., and R. M. Thorne (1998b), Electron scattering loss in Earth's inner magnetosphere: 2. Sensitivity to model parameters, J. Geophys. Res., 103(A2), 2397-2407.

Bamber, J. F., J. E. Maggs, and W. Gekelman (1995), Whistler wave interaction with a density striation: A laboratory investigation of an auroral process, J. Geophys. Res., 100(A12), 23,795-23,810.

Bell, T. F., and H. D. Ngo (1990), Electrostatic lower hybrid waves excited by electromagnetic whistler mode waves scattering from planar magneticfield-aligned plasma density irregularities, J. Geophys. Res., 95(A1), $149-172$.

Berthelier, J.-J., M. Malingre, R. Pfaff, E. Seran, R. Pottelette, J. Jasperse, J.-P. Lebreton, and M. Parrot (2008), Lightning-induced plasma turbulence and ion heating in equatorial ionospheric depletions, Nat. Geosci., $1,101-105$.

Borisov, N. D. (1995), Transformation of VLF electrostatic waves into whistlers under the action of strong HF radio waves, Phys. Lett., A, 206, 240-246.

Deng, X. H., and H. Matsumoto (2001), Rapid magnetic reconnection in the Earth's magnetosphere mediated by whistler waves, Nature, 410, 557560.

Djuth, F., et al. (1985), Observations of E region irregularities generated at auroral latitudes by a high-power radio wave, J. Geophys. Res., 90, $12,293-12,306$

Gushchin, M. E., S. V. Korobkov, A. V. Kostrov, A. V. Strikovsky, and T. M. Zaboronkova (2005), Propagation of whistlers in a plasma with a magnetic field duct, Pis'ma Zh. Eksp. Teor. Fiz., 81, 274-277. (JETP Lett., Engl. Transl., 81, 214-217)

Inan, U. S., and T. F. Bell (1991), Pitch angle scattering of energetic particles by oblique whistler waves, Geophys. Res. Lett., 18(1), 49-52.

Inan, U. S., T. F. Bell, J. Bortnik, and J. M. Albert (2003), Controlled precipitation of radiation belt electrons, J. Geophys. Res., 108(A5), 1186, doi:10.1029/2002JA009580.

Kelley, M. C., T. L. Arce, J. Salowey, M. Sulzer, W. T. Armstrong, M. Carter, and L. Duncan (1995), Density depletions at the 10-m scale induced by the Arecibo heater, J. Geophys. Res., 100(A9), 17,367-17,376.

Lee, M. C., and S. P. Kuo (1984), Production of lower hybrid waves and field-aligned plasma density striations by whistlers, J. Geophys. Res., 89, $10,873-10,880$

Minkoff, J. (1974), Radio frequency scattering from a heated ionospheric volume, 3, cross section measurements, Radio Sci., 9(11), 997-1004.

Minkoff, J., P. Kugelman, and I. Weissman (1974a), Radio frequency scattering from a heated ionospheric volume: 1. VHF/UHF field aligned and plasma-line backscatter measurements, Radio Sci., 9(11), 941-955.

Minkoff, J., M. Laviola, S. Abrams, and D. Porter (1974b), Radio frequency scattering from a heated ionospheric volume: 2 . Bistatic measurements, Radio Sci., 9(11), 957-963.

Moullard, O., A. Masson, H. Laakso, M. Parrot, P. Décréau, O. Santolik, and M. Andre (2002), Density modulated whistler mode emissions observed near the plasmapause, Geophys. Res. Lett., 29(20), 1975, doi:10.1029/2002GL015101.

Rosenberg, S., and W. Gekelman (1998), Electric field measurements of directly converted lower hybrid waves at a density striation, Geophys. Res. Lett., 25(6), 865-868.

Rosenberg, S., and W. Gekelman (2000), A laboratory investigation of lower hybrid wave interactions with a field-aligned density depletion, Geophys. Res. Lett., 27(6), 859-862.

Rosenberg, S., and W. Gekelman (2001), A three-dimensional experimental study of lower hybrid wave interactions with field aligned density depletions, J. Geophys. Res., 106(A12), 28,867-28,884.

Streltsov, A. V., M. Lampe, W. Manheimer, G. Ganguly, and G. Joyce (2006), Whistler propagation in inhomogeneous plasma, J. Geophys. Res., 111, A03216, doi:10.1029/2005JA011357.

Thome, G. D., and D. W. Blood (1974), First observations of RF backscatter from field-aligned irregularities produced by ionospheric heating, Radio Sci., 9(11), 917-921.

Vas'kov, V. V., N. I. Bud'ko, O. V. Kapustina, M. Yu Mikhailov, N. A. Ryabova, G. L. Gdalevich, G. P. Komrakov, and A. N. Maresov (1998), Detection on the Intercosmos-24 satellite of VLF and ELF waves stimulated in the topside ionosphere by the heating facility "Sura", J. Atmos. Sol.-Terr. Phys., 60, 1261-1274.

Zaboronkova, M. T., A. V. Kostrov, A. V. Kudrin, S. V. Tikhonov, A. V. Tronin, and A. A. Shaikin (1992), Channeling of whistler-range waves in inhomogeneous plasma structures, Zh. Eksp. Teor. Fiz., 102, 1151-1166. (Sov. Phys. JETP, Engl. Transl., 75, 625-632)

B. Eliasson, Department of Physics, Umeå University, SE-901 87 Umeå, Sweden. (bengt@tp4.rub.de)

K. Papadopoulos, Departments of Physics and Astronomy, University of Maryland, College Park, MD 20742-2421, USA. (dpapadop@umd.edu) 